\title{
Subcontract
}

HDE-AC03-89ER-51114

UCRL- -21233

DE90 008348

\section{POWER THRESHOLD FOR NEUTRAL BEAM CURRENT DRIVE}

\author{
P. A. Politzer \\ General Atomics \\ San Diego, CA \\ G. D. Porter
}

Lawrence Livermore National Laboratory

Livermore, CA

October, 1989

\section{DISR.LAIMER}

This report was prepared as an account of work sponsured by an agency of the United States Government. Neither the United Slates Government nor any agency Lhereaf, nor any of their cmployess, makes any wurranty, express or implied, or assumes any legal liability or respmis:bility for the accuracy, completeness, or usefulness of any information, apparatus, product, of process disclosed, or represents that its use would not infringe privately owned riglits. Reference herein to any specific commercial produch, process, ot service oy trade name, qrademark. manufactures, or otherwise does not necessarily constitule or imply is endorsement, tecommendation, ur favoring by the United States Government or any ageacy thereof. The vinws and opinions of authors exptessed herein do not necessarily state or reflacl those of the United States Government or any agency thereor. 
Work performed under the wuspices of the $L$ ist Departmeal of feorgy by the Lampence tivermare l.abotatory under cunttace number W.7405-1.X(i-4k.

This document was prepared an an account of nork upontored by an apency of the l'aited Sigles Ciovern. ment. Seithat the I'niled Stales Ciovernment ner ang upency thereof, aor any of their employerw, makes any warranty, expressed or implied, or asswines nat legal liability or responsibility for the gccuracy, complescness, of usefulness of any information. apparatus. produci. of prosess disclosed, of represents thal its use nould not infringe prisutely orned sights. Reference hertin to any sperific commezcial product, process, us service by Irade name. Irudemark, munufacluser, or otherwise, does not necesturily constituls or imply its endarscment, recommendation, ar faroring by the $\mathrm{l}$ initod states foreznment or any apenty theresf. The virws and opiniom of authurs exprested herein do not neceswarily state or rellect thow' of th: I nited States Ciosernm at ur uny agenes thetur. 


\title{
Power Threshold for Neutral Beam Current Drive
}

\author{
P. A. Politzer and G. D. Porter"
}

General Atomics, San Diego CA

\section{Abstract}

For fully noninductive current drive in tokamaks using neutral beams, there is a power and density threshold condition, setting a minimum value for $P^{9 / 2} / n^{2}$. If this condition is not met, a stationary state cannot occur, and a tokamak discharge will collapse. This is a consequence of the coupling between current and electron temperature, or between current drive efficiency and energy confinement time.

This note is intended to point out a potentially important consideration in the development of tokanaks based on fully noninduetive current drive, particularly for designs that contemplate noninductive curent buildup. The analysis has been motivated by the observation of slowly collapsing discharges in the. DIII-D tokamak during fully noninductive neutral beam current drive (NBCD) experiments. Under some conditions in these discharges, after establishment of a state in which both the thermal energy and the toroidal current are provided by the neutral beams, both the current and the thermal energy decay with a characteristic time of a few seconds (see Figure 2). This appears to be a direct consequence of the coupling which resuits from the current-dependence of the energy confinement time and the dependence of the current drive efficiency on temperature. Because the threshold falls in the parameter range of interest for presen" experiments and future designs, it is important to take this phenomenon into account.

The important features of this process can be extracted from a zero-dimensional model. For simplicity, assume that the density $n=\left\langle n_{e}(r)\right\rangle$ is independent of time. Also take equal electron and ion temperatures, $\left(T_{e}\right\rangle=\left\langle T_{i}\right\rangle=T$. The thermal energy content of the plasma is determined by

$$
3 n \frac{d T}{d t}=-\frac{3 n T}{\tau E}+\frac{P}{e V}
$$

\footnotetext{
- Lawrence Livermore National Laboratory, Livermore, CA.
} 
where $\tau_{E}$ is the global energy confinement time, $V$ is the volume, and the deposited power is $P$. All units are SI, except that the temperature is measured in $\mathrm{eV}$. The power $P$ is assumed to go to both heating and current drive. The evolution of the plasma current is given by

$$
\tau_{L / R} \frac{d I}{d t}=-I+\frac{P \eta_{C D}}{n R}
$$

where $\tau_{L / R}$ is the magnetic time constant, $R$ is the major radius, and $\eta_{C D}$ is the current drive figure of merit $\left(=n I_{C D} R / P_{C D}\right)$.

The magnetic time constant is the ratio of the inductance to resistance of the plasma, or approximately

$$
\tau_{L / R}=k_{L / R} T^{3 / 2}
$$

where

$$
k_{L / R} \simeq \frac{\mu_{0} \kappa a^{2}\left(\ln \frac{8 R}{a}-2+\frac{\ell_{i}}{2}\right)}{2\left(\eta_{0} T_{0}^{3 / 2}\right)}
$$

Here, $\kappa$ is the plasma elongation, $a$ is the minor radius, $\ell_{i}$ is the internal inductance, and $\eta_{0}$ is the resistivity at temperature $T_{0}$. Typically, in large machines the inductance is of the order of a few microhenries, the resistance is a few times $10^{-8}$ ohms, and $T_{L / \Omega}$ falls in the range of tens to hundreds of seconds.

For the energy confinement time there are a wide variety of possible choices. For this analysis we use Goldston's scaling ${ }^{1}$ because it provides an adequate fit to large tokamak data, contains the important dependences on current and power, and has a simple form tseful for analytic manipulation:

$$
\tau_{E}=k_{E} \frac{I}{P^{1 / 2}}
$$

where

$$
k_{E} \simeq 3.3 \times 10^{-5} C_{E} \frac{\kappa^{1 / 2} A_{i}^{1 / 2} R^{1.75}}{a^{0.37}}
$$

$A_{\mathrm{i}}$ is the plasma ion atomic mass, and $C_{E}$ is a multiplier to account for possible con. finement enhancement (the NBCD experiments on the DIII-D tokamak show $C_{E} \sim 1$ ). We note that the quajitative conclusions presented here regarding threshold behavior do not depend on the particular choice of confinement scaling rule. Any scaling relation for which $\tau_{E}$ improves with $I$ and worsens with $P$ will lead to similar behavior.

1 R. J. Goldston, Plasma Physics and Controlled Fusion 2887 (1984). 
Typically, $\tau_{E}$ is a fraction of a second, much smaller than $\tau_{L / R}$, and so the temperature is always in quasi-equilibrium on the time scale of the current evolution:

$$
\frac{3 n T}{\tau_{E}}=\frac{P}{e V}
$$

If, as with the Goldston scaling, $\tau_{E}$ is not an explicit function of temperature, this can be soived for

$$
T=\frac{1}{3 e V} \frac{P_{T E}}{n}
$$

Neutral beams are the source for noninductive current drive in the cases discussed here. However, it should be noted that a power threshold will also occur for any other current drive scheme that shows similar dependence on electron temperature. The second term in Eqn. 2 is the beam-driven current, $I_{C D}$. This is obtained by averaging the local (one-dimensional) current over the plasma profiles. The local current is

$$
J_{C D}=J_{\text {circ }}\left[1-\frac{Z_{b}}{Z_{x / 1}}(1-G)\right]
$$

The first term is the circulating fast ion current, and the second is the electron response, including finite aspect ratio effects in $G\left(Z_{e} f f, \epsilon\right)$. Note that the electron correction depends on beam charge $\left(Z_{b}\right)$, average ion charge $\left(Z_{e f f}\right)$, and inverse aspect ratio $(\epsilon)$, and can be treated as a simple multiplier of the fast ion current: $J_{C D}=J_{\text {circ }} F$. There is a weak dependence on temperature because the trapping factor $G$ depends on the collisionality regime, but this is ignored. The factor $G$ has been calculated by Start and Cordey for the collisionless regime, and their numerical results are well approximated by $G \simeq\left(1.55+0.85 / Z_{\text {eff }}\right) \sqrt{\epsilon}-\left(0.20+1.55 / Z_{\mathrm{e} f f}\right) \epsilon$.

The fast ion current is

$$
J_{\text {circ }}=e Z_{b} S_{b} \tau_{1} v_{b 0} \xi_{0} I(x, y)
$$

where $S_{b}=P / e^{\mathrm{t} / E_{b 0}}$ is the source strength ( $E_{b 0}$ is the injected beam energy), $\tau_{\mathrm{s}}=$ $\left[\left(4 \pi \varepsilon_{0}\right)^{2} 3 m_{e} m_{b} v_{i e}^{3}\right] /\left[16 \sqrt{\pi} e^{4} n_{e} Z_{b}^{2} \ln \Lambda\right]$ is the slowing-down time, $v_{b 0}$ is the injected beam velocity, and $\xi_{0}$ is the cosine of the angle between the injected beam and the toroidal direction. The distribution of fast ions is accounted for in the integral

${ }^{2}$ D. F. H. Start and J. G. Cordey, Phys. Fluidd 231477 (1980). 
$I(x, y)=\frac{1}{v}\left(\frac{1+z^{3}}{z^{3}}\right)^{y} \int_{0}^{z}\left(\frac{u^{3}}{1+u^{3}}\right)^{y+1} d u$. Mikkelsen and Singer ${ }^{3}$ give an approximate fit to this function: $I(x, y) \approx x^{3} /\left[(4+3 y)+\left(1.39+0.61 y^{0.7}\right) x^{2}+x^{3}\right]$. The arguments are $x=v_{b 0} / v_{c}$, where $v_{\varepsilon}=v_{t c}\left[(3 \sqrt{\pi} / 4)\left(m_{\varepsilon} / m_{b}\right)\left(Z_{e f /} / Z_{2}\right)\right]^{1 / 3}$ is the velocity at which equal momentum goes to electrons and ions, and $y=Z_{2} / 3$. The mass-weighted charge factor is $Z_{2}=\left(\sum n_{i} Z_{i}^{2}\right) /\left(m_{b} \sum n_{i} Z_{i}^{2} / m_{i}\right)$.

Defining $T_{c}$ as the temperature at which $v_{c}=v_{b 0}$ gives $x=\left(T_{c} / T\right)^{1 / 2}$, with $T_{c} \simeq 6.76 \times 10^{-2} A_{b}^{-1 / 3}\left(Z_{2} / Z_{e f f}\right)^{2 / 3} E_{b 0}$. Putting all of this together, and averaging over the plasma cross-section gives

$$
I_{C D}=k_{C D} \frac{P T^{3 / 2}}{n} \frac{\left(T_{c} / T\right)^{3 / 2}}{c_{1}+c_{2}\left(T_{v} / T\right)+\left(T_{c} / T\right)^{3 / 2}}
$$

with

$$
k_{C D}=1.39 \times 10^{18} \frac{A_{b}^{1 / 2} \xi_{0} F}{Z_{b} \ln \Lambda} \frac{1}{R E_{b 0}^{1 / 2}}
$$

where $c_{1}=4+3 y$ and $c_{2}=1.39+0.61 y^{0.7}$. Substitution of $I_{C D}$ in Eqn. 2 gives

$$
k_{L / R} T^{3 / 2} \frac{d I}{d t}=-I\left[1-k_{C D} \frac{P T^{3 / 2}}{I n} \frac{1}{c_{1}\left(T / T_{c}\right)^{3 / 2}+c_{2}\left(T / T_{c}\right)^{1 / 2}+1}\right]
$$

The possibility of a threshold is clear from this expression. If the relationship between $T$ and $I$ (determined by the energy confinement time scaling) is such that there is a maximum in the second term in brackets, then a stationary state can only exist if the value of that maximum exceeds 1 .

Define norroalized current and temperature variables, $u=I / I$. and $v=T / T$., where

$$
I_{*}=k_{C D} \frac{T_{c}^{3 / 2}}{c_{1}} \frac{P}{n} \quad \text { and } \quad T_{*}=\frac{k_{E}}{3 e V} k_{C D} \frac{T_{c}^{3 / 2}}{c_{1}} \frac{P^{3 / 2}}{n^{2}}
$$

Eqn. 8 gives the temperature in terms of the plasma current as simply

$$
v=v
$$

and the time dependence is given by

$$
\frac{d u}{d \hat{t}}=-\frac{1}{u^{1 / 2}}+\frac{1}{u^{3 / 2}+\alpha u^{1 / 2}+\beta}
$$

3 D. R. Mikkelsen and C. E. Singer, Nucl. Technology/Fusion 4237 (1983). 
where $\hat{t}=t / k_{L / R} T_{*}^{3 / 2}, a=\left(c_{2} / c_{1}\right)\left(T_{c} / T_{*}\right)$, and $\beta=\left(1 / c_{1}\right)\left(T_{c} / T_{*}\right)^{3 / 2}$.

First of all, consider possible stationary solutions to Eqn. 16. The right-hand side of this equation is sketched in Figure 1. Note first that, at low $P^{\$ / 2} / n^{2}\left(T_{.} / T_{c} \ll 1\right)$ there are no stationary solutions. ( $d I / d t$ ) is always negative, and the plasma collapses to zero current and temperature for any initial values. At higher $P^{3 / 2} / n^{2}$ there are two stationary solutions. Only the one at higher $I$ and $T$ is stable. If the initial current is below the lower stationary point, the plasma will collapse even is sufficient power is applied. Thus, there is a threshold condition on $P^{3 / 2} / n^{2}$ for the existence of a stationary state, and also a minimum initial current required for reaching this state via noninductive current build-up. The existence of a minimum current for any power level has important implications for the design of tokamaks that start up with no inductive drive.

Figure 2. Schematic of $d u / d t$ versus $u$ (Equation 16) for low, threshold, and nigh values of $P^{3 / 2} / n^{2}$. Arrowheads indicate the direction in which the system state moves.

The steady-state solution of Eqn. 16 is given by the roots of

$$
0=u^{3 / 2}+(\alpha-1) u^{1 / 2}+\beta
$$

These roots can be found analytically. If the quantity $\nu=(\alpha-1)^{3} / 27+\beta^{2} / 4$ is positive, there is one real root which is non-physical $\left(u^{1 / 2}<0\right)$. If $\nu$ is negative, there are two positive real roots. The threshold condition is $\nu \leq 0$, giving $T$. $/ T_{\mathrm{c}} \geq C_{Z} / \mathrm{c}_{1}$ 
or

$$
\frac{P^{3 / 2}}{n} \geq \frac{C_{Z}}{\left(k_{E} / 3 \mathrm{eV}\right) k_{C D} T_{c}^{1 / 2}}
$$

where $C_{Z}=c_{2}+3\left(c_{1} / 4\right)^{1 / 3}=1.39+0.61\left(Z_{2} / 3\right)^{0.7}+3\left(1+Z_{2} / 4\right)^{1 / 3}$. In terms of the beam, plasma, and machine parameters, this becomes

$$
\frac{P^{3 / 2}}{n^{2}} \geq 7.96 \times 10^{-31} \frac{Z_{b}}{A_{b}^{1 / 3} \xi_{0}}\left(\frac{Z_{E f I}}{Z_{2}}\right)^{1 / 3} \frac{C_{Z} \ln \Lambda}{C_{E} A_{i}^{1 / 2} F} \kappa^{1 / 2} R^{0.25} a^{2.37}
$$

Note that this condition depends most strongly on the size of the plasma, and is independent of beam energy. Well above the thresbold, the stationary solution approaches $u=1$, or

$$
\begin{gathered}
I=I_{a}=2.44 \times 10^{18} \frac{E_{b 0} \xi_{0}}{Z_{b}} \frac{Z_{2}}{Z_{\text {eff }}\left(4+Z_{2}\right)} \frac{F}{\ln \Lambda} \frac{P}{n R} \\
T=T_{*}=5.08 \times 10^{34} \frac{E_{b 0} \xi_{0}}{Z_{b}} \frac{Z_{2}}{Z_{\mathrm{e} f f}\left(4+Z_{2}\right)} \frac{F}{\ln \Lambda} \frac{P^{3 / 2}}{n^{2} R V}
\end{gathered}
$$

Figure 2 shows the time behavior of two NBCD discharges in DIII-D that are near the power threshold. In these discharges the target plasma is prepared inductively, but the inductive drive is removed at the same time the beams are turned on. Figure $\mathbf{2 a}$ shows the plesma current and $2 \mathrm{~b}$ shows the time behavior of $P^{3 / 2} / n^{2}$. Shot 57456 is slightly above the threshold, and the current remains approximately constant from the end of the initial transient at $1250 \mathrm{~ms}$ to the decrease in injected neutral beam power at $2050 \mathrm{~ms}$. The value of $P^{3 / 2} / n^{2}$ for shot 61539 is approximately iwo-thirds that of shot 57456 , and the current decays throughout the beam pulse - slowiy at first, then more rapidly as the temperature falls. The temperature shows a similar time dependence.

In Figure 3 we summarize the results of a number of fully noninductive NBCD attempts. The measured value of $P^{3 / 2} / n^{2}$ is plotted versus the calculated threshold value (Equation 19). The coefficient $C_{E}$ varied between 0.6 and 1.2 for these plasmas, and was determined by fitting the measured confinement data to Equation 5 .

This phenomemon may be inportant because the thresholl conditions for power, density and current fall in the range of both present NBCD experiments and future large tokanak designs. Figure 4 shows the stable and unstable stationary conditions of plasma current versus applied power, for parameters characteristic of DIII-D and ITER. The corresponding temperature curves have qualitatively similar behavior, 
Laaft - NBCD Threshold - 2 October 1989

Figure 2. Time history of (a) plasma current and (b) $P^{3 / 2} / n^{2}$ for two DIII-D discharges: 57456 (solid line) and 61539 (dashed line).

Figure 3. Experimental values of $p^{3 / 2} / \pi^{2}$ versus the threshold values predicted by Equation 19 for the experimental conditions.

except that the asymptotic dependence for large power is $T \propto P^{3 / 2}$, whereas the current follows $I \propto P$ (see Equations 20 and 21). For example, for ITER at $n=$ $6 \times 10^{19} \mathrm{~m}^{-3}$, the minimum power is about $120 \mathrm{MW}$, and the current and temperature at the threshold are $13.8 \mathrm{MA}$ and $11.9 \mathrm{keV}$ respectively (this temperature does not depeud on density). 
Draft - NBCD Threshold - 2 October 1989

Figure 4, Steady-state current versus power with density as a parameter for (a) DIII-D and (b) ITER conditions. The dashed line indicates the unstable branch. The parameters are for DIII-D: $a=0.63, R=1.65, \kappa=1.7, Z_{e f f}=2.0, \ln \Lambda=18, C_{E}=2.0, A_{i}=2, A_{b}=2$, $\xi_{0}=0.71 . E_{b 0}=75 \mathrm{keV} ;$ for ITER: $a=2.2 . R=5.8, k=1.8, z_{\text {eff }}=1.5, \ln \Lambda=19, C_{E}=2.0$, $A_{i}=2.5, A_{b}=2, \xi_{0}=0.87, E_{60}=1 \mathrm{MeV}$.

An important element not included in this calculation is tiee bootstrap current. The bocistiap current is dependent on the details of the density and temparature profiles, as well as on the collisionality of the electrons. Thus it is awkward to include it in a zero dimensional formulation such as this. If included, a term proportional to $T / I$ with a factor accounting for profiles and collisionality should be added to the right-hand side of Eqn. 2. The bootstrap effect will also appear as a positive term, proportional to $u^{-3 / 2}$ on the right-hand side of Eqn. 10, with a correction for collisionality which change: the dependence of this teran to $u^{1 / 2}$ at small $u$. The behavior of this effect is qualitatively similar to the current drive term, and inclusion of the bootstrap current will not remove the threshold but will only modify the threshold conditions. 


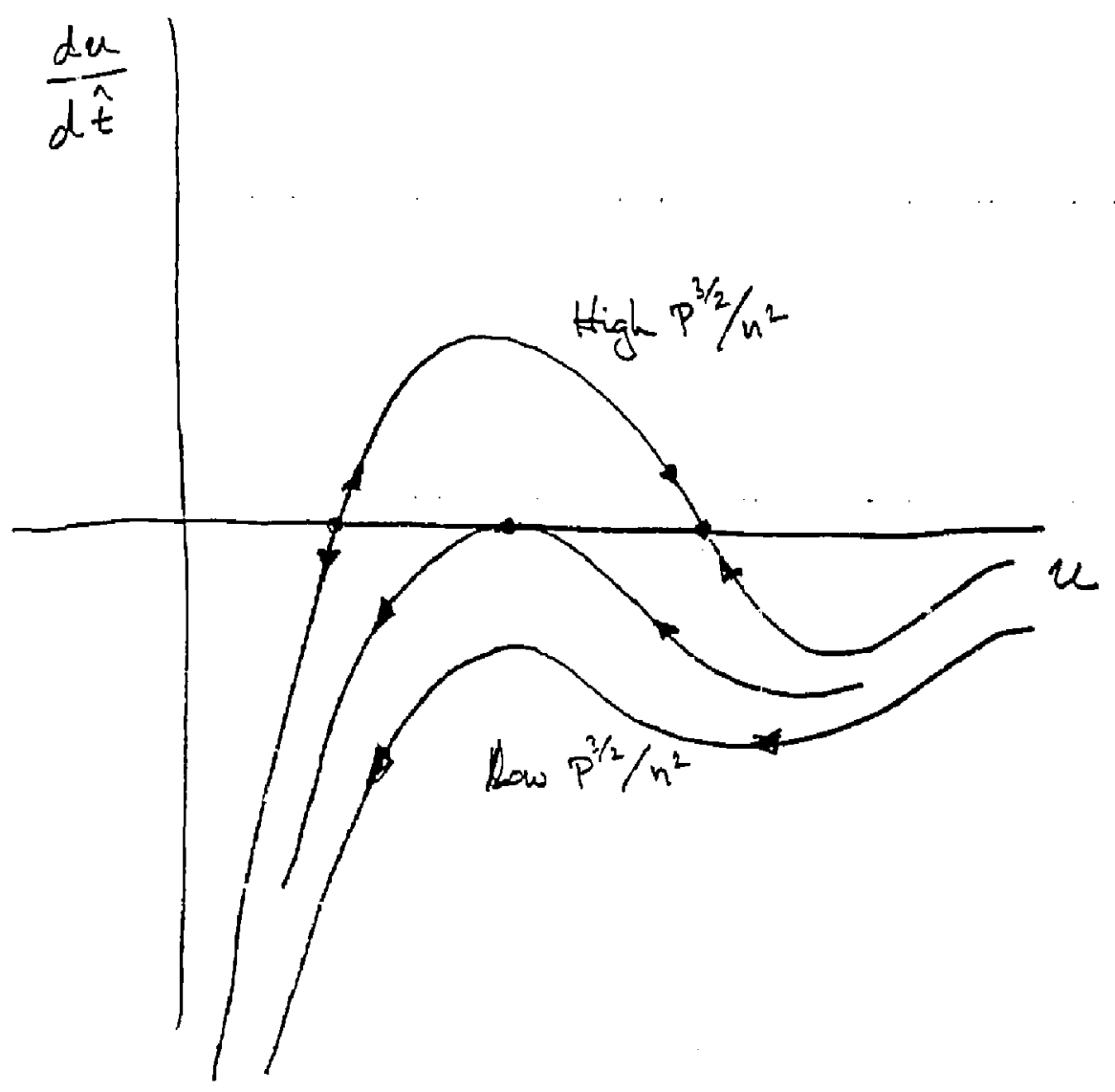


27-SEP-B9 14:02:45
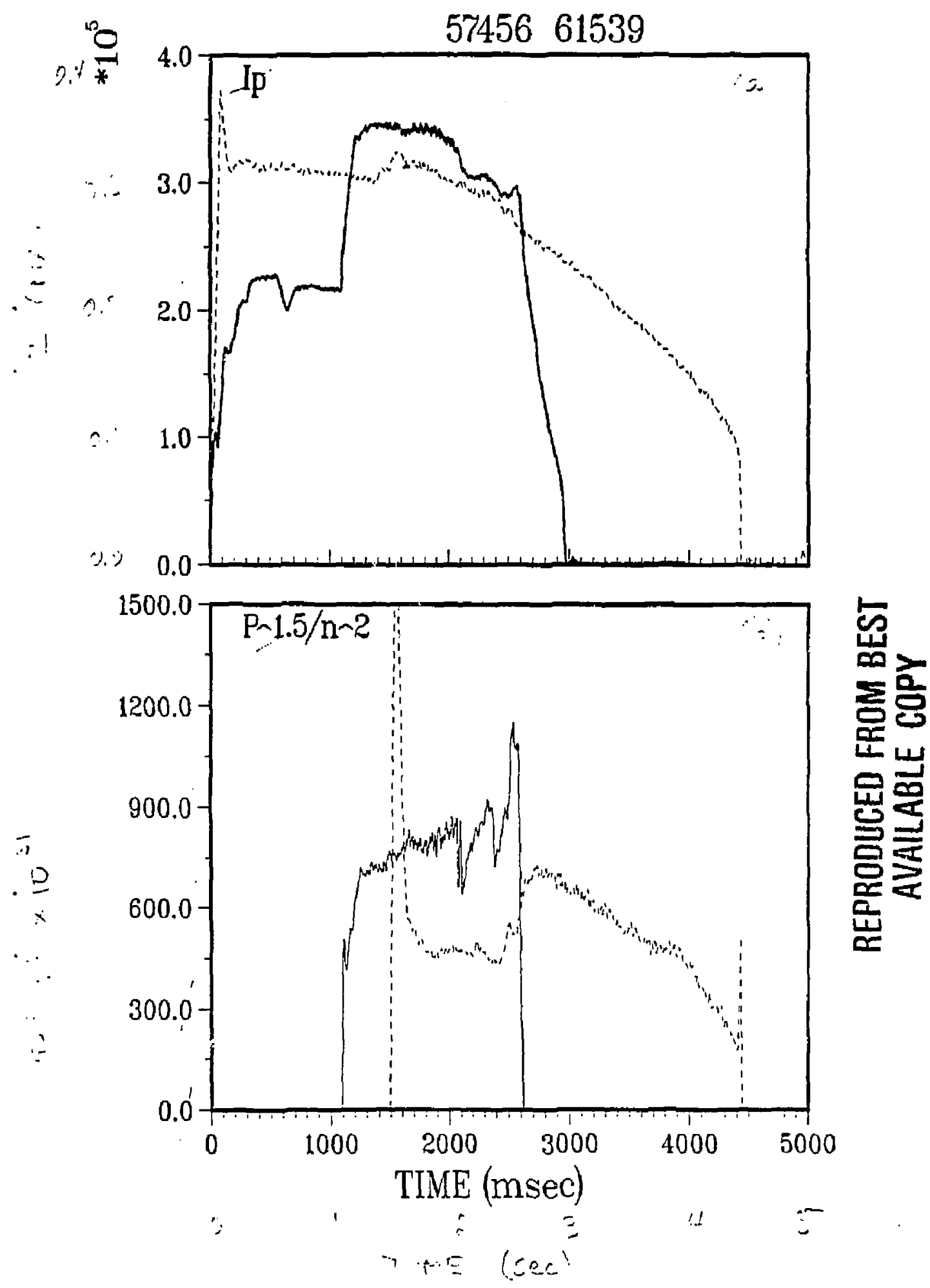

$\therefore n n d i$ 


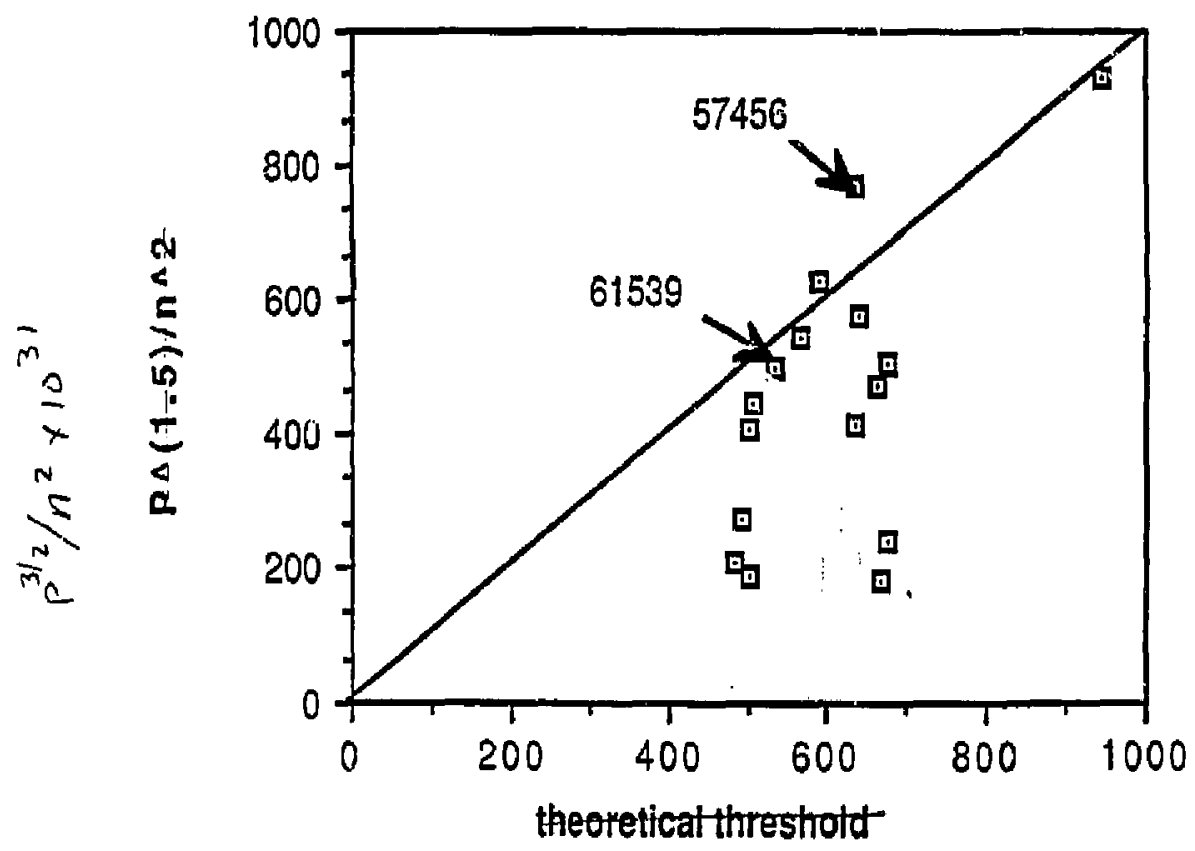

$\frac{C_{z}}{\left(\frac{k_{1}}{3_{0}}\right) k_{C D} T_{C}^{1 / 2}} \times 10^{31}$ 


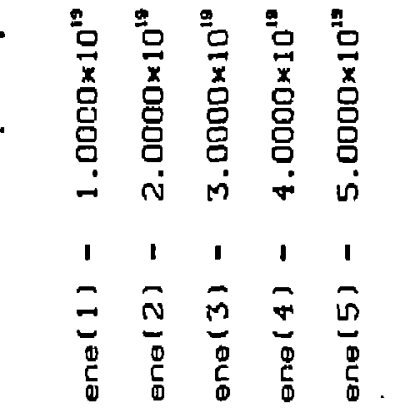
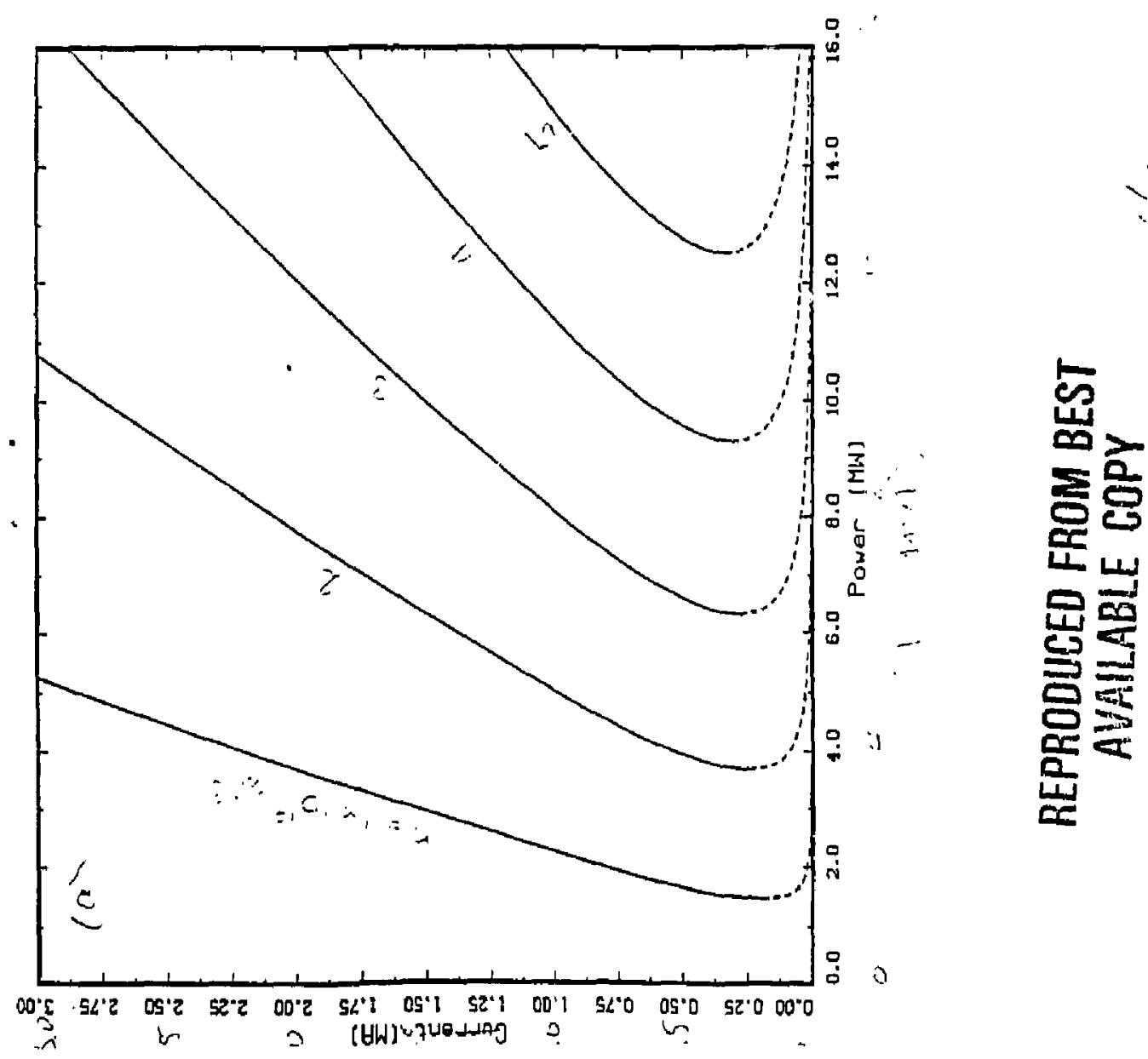

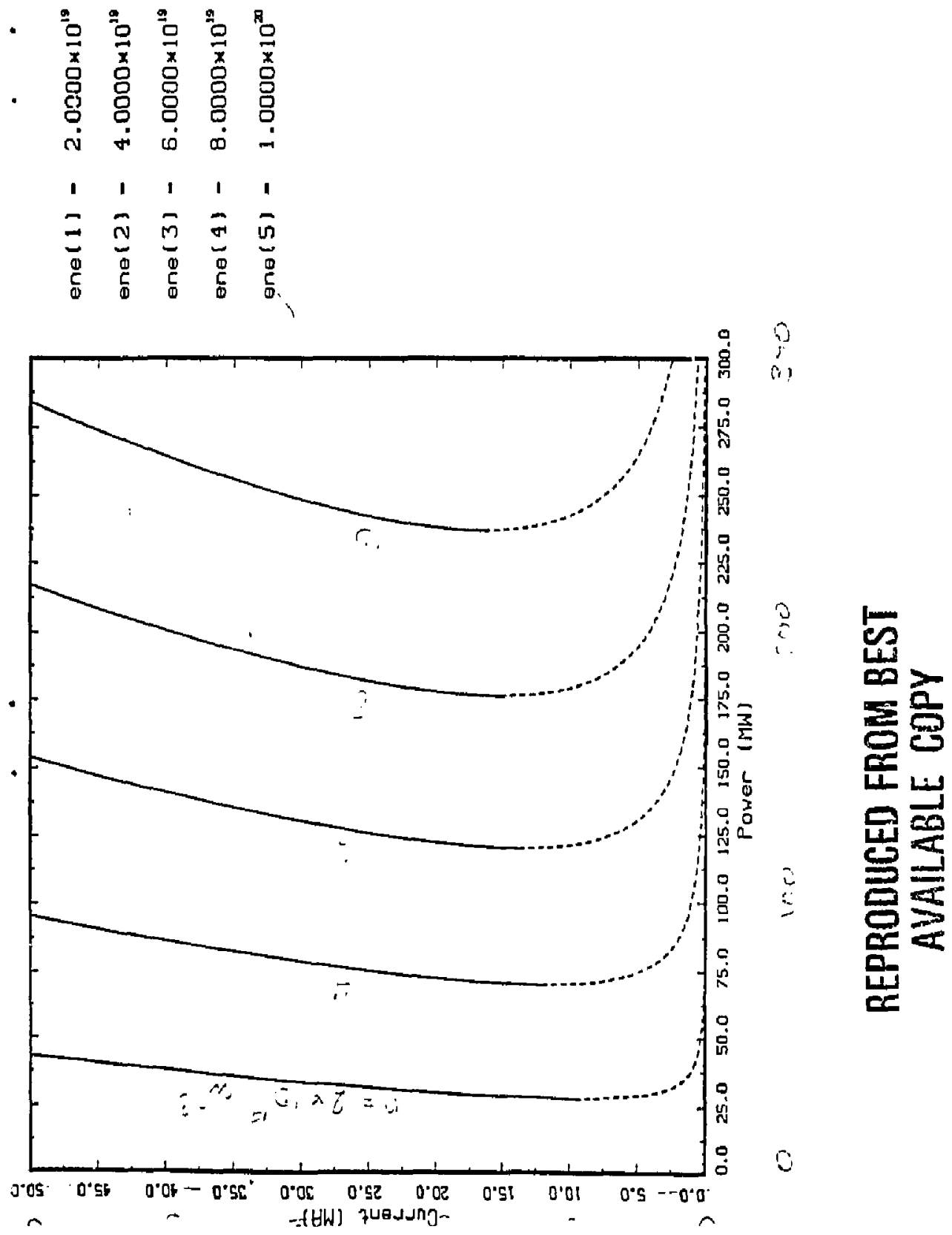